\title{
Olson's exploitation hypothesis in a public good economy: a reconsideration
}

\author{
Wolfgang Buchholz ${ }^{1,2} \cdot$ Todd Sandler ${ }^{3}$
}

Received: 4 April 2016/Accepted: 22 June 2016/Published online: 4 July 2016

(C) The Author(s) 2016. This article is published with open access at Springerlink.com

\begin{abstract}
Since the publication of Olson's (1965) The Logic of Collective Action, the exploitation hypothesis, in which the rich shoulder the provision burden of public goods for the poor, has held sway despite empirical exceptions. To address such exceptions, we establish two alternative exploitation hypotheses based on contributors' asymmetric preferences or on their productivity differences regarding the public good. The classic hypothesis and its two variants are proven in a novel fashion. For asymmetric preferences, we also establish the exploitation hypothesis for the joint products model with private and public cobenefits. Our theoretical insights are then illustrated by some empirical examples from the field of international public goods, such as military defense and cross-border pollution.
\end{abstract}

Keywords Public goods · Exploitation hypothesis - Collective action · Asymmetric preferences

JEL Classification F53 $\cdot$ H41 $\cdot$ H87

\section{Introduction}

Beginning with Olson (1965), the "exploitation hypothesis" has been of recurrent interest in the theory of public goods (see, e.g., Boadway and Hayashi 1999; Cornes and Sandler 1996; Ihori et al. 2014; Pecorino 2015; Sandler 1992). This hypothesis essentially means

Todd Sandler

tsandler@utdallas.edu

Wolfgang Buchholz

wolfgang.buchholz@ur.de

1 University of Regensburg, Regensburg, Germany

2 CESifo Munich, Munich, Germany

3 School of Economic, Political \& Policy Sciences, University of Texas at Dallas, 800 W Campbell Rd, Richardson, TX 75080, USA 
that, in a Nash equilibrium of voluntary public good provision, the rich (better endowed) agents make larger contributions to the public good than the poor agents so that the rich agents, in a certain sense, are "exploited" in the strategic context of the contribution game. However, Sandler (2015, pp. 206-209) remarked that the hypothesis can be expected to hold only under specific circumstances, i.e., if the agents behave according to the strategic Nash assumption and possess identical preferences. While maintaining the Nash assumption, we show that besides the original "exploitation of the rich by the poor" other kinds of "exploitation" are possible: i.e., of the contributors with a high preference for the public good by those with a low preference; of the contributors with high private cobenefits from their public good contributions by those with low private co-benefits; and of the contributors with greater productivity in providing the public good by those with lower productivity. Our expanded analysis of exploitation provides a more complete picture of the determinants of burden sharing with respect to voluntary public good provision. Even though differences in preferences (see Andreoni 1988) or public good productivities (see Buchholz and Konrad 1994; Ihori 1996) have been considered in the literature, these approaches have not been associated explicitly with Olson's exploitation hypothesis.

After describing the framework of the analysis in Sect. 2, we consider the three variants of the exploitation maxim under quite general assumptions in Sect. 3. In particular, we provide a short novel proof of the classical exploitation hypothesis and its two variants. In Sect. 4, we illustrate the three versions of the exploitation hypothesis through examples based on Cobb-Douglas preferences and, in particular, show how the underlying mechanisms may oppose each other. In Sect. 5, we conclude by suggesting some empirical applications of our theoretical results.

\section{The framework}

There are $n$ agents, $i=1, \ldots, n$, who are characterized by their initial private good endowments, $w_{i}$, and their utility functions, $u_{i}\left(x_{i}, G\right)$, where $x_{i}$ denotes agent $i$ 's level of private consumption and $G$ indicates public good supply. Each utility function is assumed to have the standard properties, i.e., it is twice continuously differentiable, quasi-concave, and strictly monotonically increasing in both variables. Moreover, both goods are assumed to be non-inferior for all agents. Agents may also have different constant productivities $a_{i}$ (=individual marginal rates of transformation, $m r t_{i}$ ) in providing the public good. These productivities indicate how many units of the public good agent $i$ generates if she spends one unit of the private good on the public good. The reciprocal of the productivity parameter $a_{i}$ then represents agent $i$ 's per unit cost of producing the public good.

Given the utility functions, $u_{i}\left(x_{i}, G\right)$, and public good productivities, $a_{i}$, the determination of the outcome of voluntary public good provision will essentially be based on the (income) expansion paths, $e_{i}\left(G, a_{i}\right)$, of the agents, $i=1, \ldots, n$. In $x_{i}-G$ space, agent $i$ 's expansion path connects all points $\left(x_{i}, G\right)$ at which agent $i$ 's marginal rate of substitution $m r s_{i}$ between the private and the public good is equal to $a_{i}$; i.e., $a_{i}=\frac{u_{i x}\left(x_{i}, G\right)}{u_{i G}\left(x_{i}, G\right)}$ holds, where $u_{i x}=\frac{\partial u_{i}}{\partial x_{i}}$ and $u_{i G}=\frac{\partial u_{i}}{\partial G}$ denote the two partial derivatives of the utility function. Along such an expansion path, agent $i$ 's indifference curve has slope $-a_{i}$. Non-inferiority of both goods implies that the expansion paths $e_{i}\left(G, a_{i}\right)$ are well-defined and strictly monotonically increasing functions of $G$. In order to avoid the tedious treatment of subcases, we assume that $e_{i}\left(0, a_{i}\right)=0$ and $\lim _{G \rightarrow \infty} e_{i}\left(G, a_{i}\right)=\infty$. 
For a further explanation of the expansion path concept, we note that agent $i$ standing alone and facing $p_{i}=1 / a_{i}$ as the public good price would, as a price-taker, choose public good supply $G$ and private consumption $x_{i}=e_{i}\left(G, a_{i}\right)$ if $i$ 's income were $\tilde{w}_{i}=e_{i}\left(G, a_{i}\right)+p_{i} G$. In the framework of voluntary public good provision, agent $i$ likewise would attain maximum utility at the same point $\left(x_{i}, G\right)=\left[e_{i}\left(G, a_{i}\right), G\right]$ when the other agents contribute $\tilde{G}_{-i}=G-a_{i}\left[w_{i}-e_{i}\left(G, a_{i}\right)\right]$ in total to the public good and $\tilde{G}_{-i} \leq G$ or, equivalently, $e_{i}\left(G, a_{i}\right) \leq w_{i}$ holds (see Fig. 1). If, instead, $\tilde{G}_{-i}>G$ (or, equivalently, $\left.e_{i}\left(G, a_{i}\right)>w_{i}\right)$, agent $i$ would want to make a negative contribution to the public good, which, however, is not possible. Consequently, agent $i$ attains maximum utility at a corner solution at which her public good contribution is zero.

Let $\bar{G}_{i}\left(w_{i}, a_{i}\right)$ be the level of public good supply at which the value of the expansion path, $e_{i}\left(G, a_{i}\right)$, corresponds to agent $i$ 's initial endowment $w_{i}$, i.e., $e_{i}\left[\bar{G}_{i}\left(w_{i}, a_{i}\right), a_{i}\right]=w_{i}$ holds (see Fig. 1). From our assumptions and the intermediate value theorem, it follows that $\bar{G}_{i}\left(w_{i}, a_{i}\right)$ exists, and the monotonicity of the expansion path implies that it is unique.

Given initial endowment $w_{i}$, utility functions, $u_{i}\left(x_{i}, G\right)$, and productivity parameters, $a_{i}$, it is well-known that, under the assumptions imposed above, the Nash equilibrium (NE) [denoted by $\left.\left(\hat{x}_{1}, \ldots, \hat{x}_{n}, \hat{G}\right)\right]$ of voluntary public good provision exists and is unique (Bergstrom et al. 1986; Cornes and Sandler 1996; Cornes and Hartley 2007; Fraser 1992). This NE, in which no agent can make a negative public good contribution, is characterized by the following properties.

1. The feasibility constraint $\hat{G}=\sum_{i=1}^{n} a_{i} \hat{z}_{i}$ is satisfied, where $\hat{z}_{i}=w_{i}-\hat{x}_{i} \geq 0$ is agent $i$ 's public good contribution.

2. The equilibrium position of each contributing agent $i$, i.e., for whom $\hat{z}_{i}>0$ holds, lies on $i$ 's expansion path $e_{i}\left(G, a_{i}\right)$, i.e., $\hat{x}_{i}=e_{i}\left(\hat{G}, a_{i}\right)$.

3. Agent $i$ is a contributor if and only if $\hat{G}<\bar{G}_{i}\left(w_{i}, a_{i}\right)$ (Andreoni 1988; Andreoni and McGuire 1993).

We now make use of these properties to present three different versions of the exploitation hypothesis.

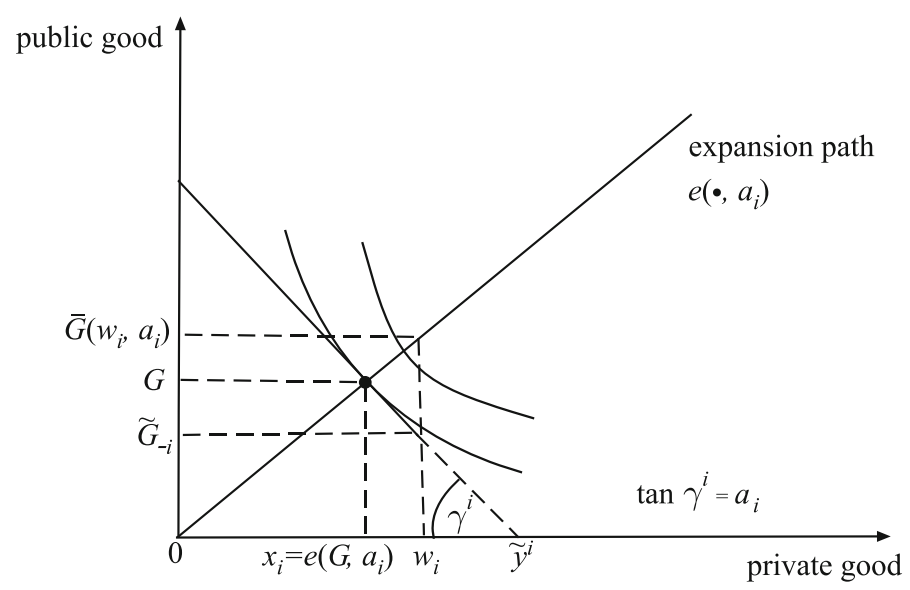

Fig. 1 Expansion path 


\section{Three versions of the exploitation hypothesis}

The three versions are obtained by assuming that the agents or contributors differ only with respect to endowment levels, or preferences, or productivities, while they are homogenous in terms of the other two properties.

Case 1 All agents have the same utility function, $u\left(x_{i}, G\right)$, and the same productivity parameter, $a$, but have different income levels, $w_{i}$.

The relevant expansion path, which is then common to all agents, is denoted by $e(G, a)$. Without loss of generality, we assume $w_{1} \leq \cdots \leq w_{n}$. The monotonicity of $e(G, a)$ thus gives $\bar{G}_{1}\left(w_{1}, a\right) \leq \cdots \leq \bar{G}_{n}\left(w_{n}, a\right)$. In Fig. 2, we, specifically, consider two agents, $j$ and $k$, with $j<k$, for whom $\bar{G}_{k}\left(w_{k}, a\right)>\bar{G}_{j}\left(w_{j}, a\right)>\hat{G}$ holds so that both are contributors to the public good (see Fig. 2).

From property (3), it follows that, at the NE, there is some $m^{*} \geq 1$ where the group of contributors consists of all rich agents for whom $i \geq m^{*}$, whereas the group of noncontributors consists of poorer agents for whom $i<m^{*}$. Furthermore, property (2) implies that, at the NE, all contributors have the same private consumption $\hat{x}=e(\hat{G}, a)$. Therefore, at the NE, the absolute levels of public good contributions of the contributing agents, $\hat{z}_{i}=w_{i}-\hat{x}$, vary positively with income $w_{i}$. This also is seen on the horizontal axis of Fig. 2. The same holds true for the relative contribution levels $\frac{\hat{z}_{i}}{w_{i}}=1-\frac{\hat{x}}{w_{i}}$, which establishes Olson's conventional exploitation maxim. Obviously, at the NE, all contributing agents attain the same utility, $u(\hat{x}, \hat{G})$, whereas non-contributors attain a smaller utility, $u\left(w_{i}, \hat{G}\right)$, ranked by income.

Case 2 All agents have the same income, $w$, and the same public good productivity, $a$, but possess different utility functions, $u_{i}\left(x_{i}, G\right)$.

In particular, we assume that agents can be ordered according to their marginal willingness to pay for the public good. At each $(x, G)$, we have $m r s_{j}=\frac{u_{j x}(x, G)}{u_{j G}(x, G)} \geq \frac{u_{k x}(x, G)}{u_{k G}(x, G)}=m r s_{k}$ if $j, k \in\{1, \ldots, n\}$ with $j<k$. The indifference curves of an agent with a lower rank $j$ are thus everywhere steeper than the indifference curves of an agent with a higher rank $k$, so that agent $k$ has a greater relative preference for the public good than that of agent $j$. Then, convexity of indifference curves implies that $e_{j}(G, a) \geq e_{k}(G, a)$ for any $G$ if $j<k$ and thus $\bar{G}_{1}(w, a) \leq \cdots \leq \bar{G}_{n}(w, a)$ (see Fig. 3).

Fig. 2 Classical income-based exploitation hypothesis

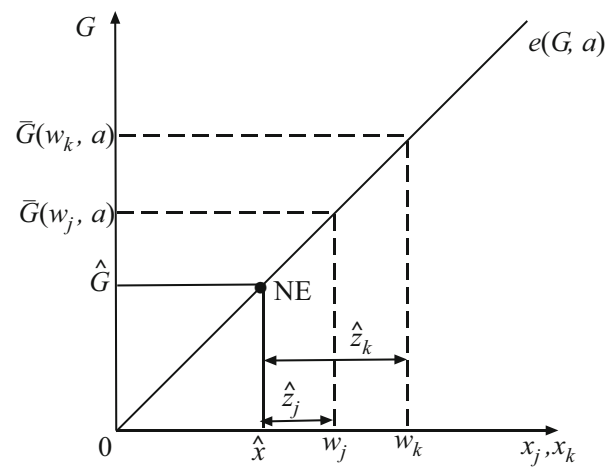


Fig. 3 Exploitation based on taste differences

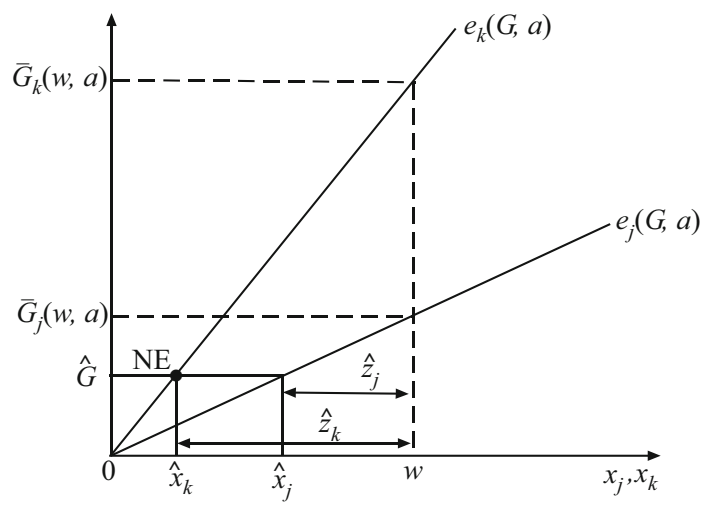

Property (3) shows that there is a $m^{*} \geq 1$ for which only agents $i \geq m^{*}$ contribute to the public good, while agents with less interest in the public good than agent $m^{*}$ contribute nothing at the NE. For contributing agents $j$ and $k$, we have, as depicted in Fig. 3, that $\hat{x}_{j}=e_{j}(\hat{G}, a) \geq e_{k}(\hat{G}, a)=\hat{x}_{k}$ and hence $\hat{z}_{j}=w-\hat{x}_{j} \leq w-\hat{x}_{k}=\hat{z}_{k}$ if $j<k$. This shows that, ceteris paribus, agents with the greater preference for the public good spend more on it at the NE in absolute and, as income $w$ is the same for all agents here, also in relative terms.

In particular, different preference intensities for the public good may be caused by private co-benefits from individual public good contributions (see, e.g., Cornes and Sandler 1994), which may either stem from some warm-glow feeling of contributing to a charitable organization (see Andreoni 1990) or from the reduction in locally damaging pollutants of contributing to a global public good (carbon dioxide mitigation) (see, e.g., Pittel and Rübbelke 2008). For this application of our analysis to impure public good models, we assume that all agents have the same "basic" utility function, $v\left(x_{i}, G\right)$, with standard properties and, as above, the same initial endowment, $w$. The ancillary benefits of their individual public good contributions, however, may be of different strength. Specifically, the private co-benefit that flows from agent $i$ 's public good contribution $z_{i}=w_{i}-x_{i}$ is measured by $\beta_{i} h\left(z_{i}\right)$, where $h\left(z_{i}\right)$ is a differentiable, strictly monotonically increasing and concave function of $z_{i}$ and $\beta_{i} \geq 0$ is an agent-specific scalar.

Given these assumptions for any agent, $i=1, \ldots, n$, we then construct an auxiliary utility function, $u_{i}\left(x_{i}, G\right)=v\left(x_{i}, G\right)+\beta_{i} h\left(w_{i}-x_{i}\right)$, defined for all $x_{i} \in\left[0, w_{i}\right)$ and $G \geq 0$. We assume that $u_{i}\left(x_{i}, G\right)$ is quasi-concave and that both the private and the public good are non-inferior with respect to (w.r.t.) this utility function, which ensures existence and uniqueness of an NE (see Kotchen 2007). Given $u_{i}\left(x_{i}, G\right)$, the marginal rate of substitution between the private and the public good at $\left(x_{i}, G\right)$ is $m r s_{i}=\frac{u_{i x}\left(x_{i}, G\right)}{u_{i G}\left(x_{i}, G\right)}=\frac{v_{i x}\left(x_{i}, G\right)-\beta_{i} h^{\prime}\left(w_{i}-x_{i}\right)}{v_{i G}\left(x_{i}, G\right)}$. As $h^{\prime}\left(w_{i}-x_{i}\right)>0$, this $m r s_{i}$ is falling at any $\left(x_{i}, G\right)$ if $\beta_{i}$ is increased, so that a greater value of $\beta_{i}$ indicates a stronger preference for the public good according to the general definition above. If we assume that $\beta_{1} \leq \cdots \leq \beta_{n}$, it follows from our previous reasoning that agent $k$ will contribute more to the public good than agent $j$ when $k>j$ in the ensuing NE. In this sense, agents that have higher private co-benefits from their public good contributions are exploited by agents with lower private co-benefits.

Case 3 All agents have the same income, $w$, and the same utility function, $u\left(x_{i}, G\right)$, but possess different productivity parameters, $a_{i}$. 
We assume that agents are ranked according to their productivities, i.e., $a_{1} \leq \cdots \leq a_{n}$. Then, we have for the expansion paths originating from the common utility function that $e\left(G, a_{j}\right) \geq e\left(G, a_{k}\right)$ holds at each $G$ if $j<k$ and, thus, $\bar{G}_{1}\left(w, a_{1}\right) \leq \cdots \leq \bar{G}_{n}\left(w, a_{n}\right)$. This can be depicted in a diagram that is completely analogous to Fig. 3.

By the same argument as in cases 1 and 2 above, it now follows that, at the NE, agents with a greater public good productivity, ceteris paribus, contribute more to the public good than agents with a lower productivity. When agents $j$ and $k$ make positive contributions to the public good, we have $\hat{z}_{j}=w-e\left(\hat{G}, a_{j}\right) \leq w-e\left(\hat{G}, a_{k}\right)=\hat{z}_{k}$.

For Case 3, all non-contributors' utility, $u(w, \hat{G})$, is the same at the NE. Contributors' utility, $u\left(\hat{x}_{i}, \hat{G}\right)$, is smaller, the greater their public good productivity. In relationship to Olson's exploitation hypothesis, this reflects the "curse of being more productive" in voluntary public good provision, which —in a different context and without direct relation to Olson's maxim—-has also been observed by Buchholz and Konrad (1994) and Ihori (1996).

\section{Cobb-Douglas examples}

\subsection{Illustration of the three cases}

For an illustration of our general results, we assume that there are $n=3$ agents. Agent $i$ possesses Cobb-Douglas preferences, $u_{i}\left(x_{i}, G\right)=x_{i} G^{\alpha_{i}}$, and, as above, has initial endowment $w_{i}$ and public good productivity $a_{i}$. From $m r s_{i}=\frac{u_{i x}\left(x_{i}, G\right)}{u_{i G}\left(x_{i}, G\right)}=\frac{G^{\alpha_{i}}}{\alpha_{i} x_{i} G^{\alpha_{i}-1}}=a_{i}$, we get $e_{i}\left(G, a_{i}\right)=x_{i}=\frac{G}{\alpha_{i} a_{i}}$ as the formula for the expansion paths of three agents, $i=1,2,3$. The condition that $e_{i}\left[\bar{G}_{i}\left(w_{i}, a_{i}\right), a_{i}\right]=w_{i}$ then yields $\bar{G}_{i}\left(w_{i}, a_{i}\right)=\alpha_{i} a_{i} w_{i}$ for $i=1,2,3$. The $m r s_{i}$ declines when the preference parameter $\alpha_{i}$ increases, so that-according to the definition given above-a larger $\alpha_{i}$ indicates a stronger preference for the public good.

For this specific configuration, we provide examples for each of the three cases, presented in the earlier general setting.

Case $1 \quad \alpha_{i}=a_{i}=1$ for $i=1,2,3$ and $w_{1}=1, w_{2}=2$, and $w_{3}=3$; i.e., the otherwise equal agents are ranked w.r.t. their income levels.

Under these assumptions, the NE is given by $\hat{x}_{1}=1$ and $\hat{x}_{2}=\hat{x}_{3}=\hat{G}=\frac{5}{3}$, so that agents 2 and 3 are contributors to the public good, while agent 1 is a non-contributor. In this allocation, individual public good contributions are $\hat{z}_{1}=0, \hat{z}_{2}=w_{2}-\hat{x}_{2}=2-\frac{5}{3}=\frac{1}{3}$, and $\hat{z}_{3}=w_{3}-\hat{x}_{3}=3-\frac{5}{3}=\frac{4}{3}$.

To confirm that this allocation is indeed the NE, we have to check the three conditions listed in Sect. 2: Clearly, $\hat{z}_{1}+\hat{z}_{2}+\hat{z}_{3}=0+\frac{1}{3}+\frac{4}{3}=\frac{5}{3}=\hat{G}$, which gives condition (1). Condition (2) holds for the contributing agents, $i=2,3$, since $e\left(\hat{G}, a_{i}\right)=\hat{G}=\frac{5}{3}=\hat{x}_{i}$. Condition (3) follows insofar as $\bar{G}_{1}(1,1)=w_{1}=1, \quad \bar{G}_{2}(2,1)=w_{2}=2$, and $\bar{G}_{3}(3,1)=w_{3}=3$, so that $\bar{G}_{1}(1,1)<\hat{G}<\bar{G}_{2}(2,1)<\bar{G}_{3}(3,1)$.

In this representation of Olson's classical exploitation hypothesis, agent 1 , with the lowest income, does not contribute to the public good in the NE. Among the two contributors, higher-income agent 3 makes a larger contribution than agent 2 to the public good and, thus, is "exploited." 
Case $2 w_{i}=w=1$ and $a_{i}=a=1$ for $i=1,2,3$, while $\alpha_{1}=\frac{1}{2}, \alpha_{2}=\frac{3}{4}$, and $\alpha_{3}=1$; i.e., the otherwise equal agents are ranked w.r.t. their preference intensity for the public good.

The three conditions that characterize the NE are fulfilled at $\hat{x}_{1}=1, \hat{x}_{2}=\frac{4}{5}$, and $\hat{x}_{3}=\hat{G}=\frac{3}{5}$, where the individual public good contributions are $\hat{z}_{1}=0, \hat{z}_{2}=\frac{1}{5}$, and $\hat{z}_{3}=\frac{2}{5}$, respectively. Hence, condition (1) holds. Condition (2) follows from $e_{2}(\hat{G}, 1)=\frac{\hat{G}}{\alpha_{2}}=$ $\frac{4}{3} \times \frac{3}{5}=\frac{4}{5}=\hat{x}_{2} \quad$ and $\quad e_{3}(\hat{G}, 1)=\frac{\hat{G}}{\alpha_{3}}=1 \times \frac{3}{5}=\frac{3}{5}=\hat{x}_{3} . \quad$ Given $\quad$ that $\quad \bar{G}_{1}(1,1)=\alpha_{1}=\frac{1}{2}$, $\bar{G}_{2}(1,1)=\alpha_{2}=\frac{3}{4}$, and $\bar{G}_{3}(1,1)=\alpha_{3}=1$ for these preferences, condition (3) also is satisfied.

Agent 1, with the smallest preference for the public good, is a complete free rider. Among the two contributors, agent 3 with the strongest public good preference contributes more to this good and, thus, is "exploited" by agent 2 , who possesses a smaller public good preference.

Case $3 w_{i}=\alpha_{i}=1$ for $i=1,2,3$, and $a_{1}=\frac{1}{4}, a_{2}=\frac{1}{3}$, and $a_{3}=\frac{1}{2}$.

Now, the NE corresponds to $\hat{x}_{1}=1, \hat{x}_{2}=\frac{5}{6}, \hat{x}_{3}=\frac{5}{9}$, and $\hat{G}=\frac{5}{18}$, for which individual public good contributions, as measured in the units of the private good, are $\hat{z}_{1}=0, \hat{z}_{2}=\frac{1}{6}$, and $\hat{z}_{3}=\frac{4}{9}$. For this allocation, we have: $a_{1} \hat{z}_{1}+a_{2} \hat{z}_{2}+a_{3} \hat{z}_{3}=\frac{1}{4} \times 0+\frac{1}{3} \times \frac{1}{6}+\frac{1}{2} \times \frac{4}{9}=$ $\frac{5}{18}=\hat{G}$, which gives condition (1). Condition (2) follows since $e_{2}\left(\hat{G}, \frac{1}{3}\right)=3 \times \frac{5}{18}=\frac{5}{6}=\hat{x}_{2}$ and $e_{3}(\hat{G}, 1)=2 \times \frac{5}{18}=\frac{5}{9}=\hat{x}_{3}$. Condition (3) is satisfied because $\bar{G}_{3}\left(1, \frac{1}{2}\right)=a_{3}=$ $\frac{1}{2}>\bar{G}_{2}\left(1, \frac{1}{3}\right)=a_{2}=\frac{1}{3}>\hat{G}=\frac{5}{18}$, while $\bar{G}_{1}\left(1, \frac{1}{4}\right)=a_{1}=\frac{1}{4}<\frac{5}{18}=\hat{G}$.

In this example, the most productive agent, agent 3 , spends more of her income on the public good and, thus, is "exploited" by agents 1 and 2 . Least-productive agent 1 contributes nothing to the public good.

\subsection{Interaction of the partial effects}

The partial effects that underlie the above three cases may interact and oppose each other. Either because of weak preferences for the public good or a low public good productivity, it is possible that an agent (e.g., a country) with a relatively high income contributes less to the public good than a poorer one and even may become a complete free rider. The interaction of the different partial effects can be visualized by the following examples, in which we again consider an economy with three agents, who all possess Cobb-Douglas preferences, $u_{i}\left(x_{i}, G\right)=x_{i} G^{\alpha_{i}}$, and whose income levels are $w_{1}=1, w_{2}=2$, and $w_{3}=3$.

In the first example, we assume that the three agents have the same public good productivity $a_{i}=1$, but display different preferences: i.e., $\alpha_{1}=1, \alpha_{2}=\frac{1}{3}$, and $\alpha_{3}=\frac{1}{6}$, so that agent 1 has the smallest income but the strongest preference for the public good, while agent 3 has the largest income but the weakest preference for the public good. In this situation, the NE is given by $\hat{x}_{1}=\hat{G}=\frac{3}{5}, \hat{x}_{2}=\frac{9}{5}$, and $\hat{x}_{3}=3$, which, as before, is confirmed by checking the three conditions that characterize a NE. ${ }^{1}$ The individual public good contributions thus are $\hat{z}_{1}=\frac{2}{5}, \hat{z}_{2}=\frac{1}{5}$, and $\hat{z}_{3}=0$, so that the ranking of public good contributions is reversed from that of the standard exploitation hypothesis. Owing to a strong

\footnotetext{
1 The details of the proofs for this subsection can be obtained from the authors upon request.
} 
public good preference, the poorest agent pays the most for the public good, while the richest agent, who values the public good the least, contributes nothing.

In the second example, the three agents have the same Cobb-Douglas preference with $\alpha_{i}=1$, but display different public good productivities, for which $a_{1}=1, a_{2}=\frac{1}{3}$, and $a_{3}=\frac{1}{6}$, so that agent 1 with the smallest income has the largest productivity, while agent 3 with the largest income has the smallest productivity. The NE then is given by $\hat{x}_{1}=\hat{G}=\frac{5}{9}$, $\hat{x}_{2}=\frac{5}{3}$, and $\hat{x}_{3}=3$, so that the individual public good contributions are $\hat{z}_{1}=\frac{4}{9}, \hat{z}_{2}=\frac{1}{6}$, and $\hat{z}_{3}=0$. Now, the poorest, but most productive, agent spends the most for the public good, whereas the richest, but least productive, agent takes a free ride.

These two examples show how the conventional exploitation effect may be counterbalanced by the effects that underlie the other two versions of the exploitation maxim, presented in this paper.

\section{Some empirical applications}

To highlight the relevancy of the three exploitation hypotheses, we provide some illustrations drawn from international public goods.

Before presenting these illustrations, we must review how the literature goes from the individual provision of public goods to the country provision of an international public good. Generally speaking, the literature treats the country as a unitary actor with the other contributors being countries, each of which likewise is a unitary actor (see, e.g., Boadway and Hayashi 1999; Buchholz et al. 2014; Cornes and Sandler 1996; Ihori 1996; Murdoch and Sandler 1997). As such, gross domestic product (GDP) replaces income in the budget constraint, which already had been a central assumption in Olson and Zeckhauser's (1966) seminal NATO burden-sharing study. Consistent with our paper, their underlying model is that of deterrence as a pure public good with a NE at which each ally equates its marginal rate of substitution, $\frac{\partial u_{i} / \partial x_{i}}{\partial u_{i} / \partial G}\left(x_{i}, G\right)$, to the reciprocal of the per unit price of defense since the private good's price is normalized to 1 . This approach has been taken by the literature because the capacity of a country to provide defense is better captured by its productive capacity, represented by total GDP, rather than by its average income per capita or wellbeing. ${ }^{2}$ Henceforth, we use GDP, and not per capita income, as the income measure to denote "rich," since this is the one that is primarily used by the standard literature's notion of exploitation for international public goods. For many international public goods, GDP rather than average per capita income better measures the capacity of the country to provide the good.

The first application of the classical exploitation hypothesis was to the NATO alliance, in which—despite the member states' commitment to mutual assistance-allies voluntarily contribute to collective defense. Defense spending that deters an enemy's attack on the allies constitutes the voluntary provision of a pure public good. With respect to burden sharing within the NATO, Olson and Zeckhauser (1966) hypothesized that rich allies

\footnotetext{
${ }^{2}$ For contributing and non-contributing countries, Boadway and Hayashi (1999) explored the effects of population differences on exploitation with respect to an international public good. The standard exploitation hypothesis, based on GDP, carries over to per capita income provided that population is the same among countries. If, however, population differs among countries, Boadway and Hayashi (1999, p. 624) showed that more populous contributing countries consume less of the private good and are worse off than less populous countries regardless of per capita income. Moreover, non-contributing countries are better off if their per capita income is larger.
} 
would shoulder more of the alliance's deterrence burdens than the "poor" allies, where GDP served as the income proxy. To test this exploitation hypothesis, they used the allies' military expenditures (MEs) as shares of GDP as the burden measure. The ME/GDP ratio indicates an ally's relative defense contribution in terms of its productive capacity, which is not the case for an analogous ratio based on per capita income. Using a Spearman rank correlation test, Olson and Zeckhauser (1966) found a significant positive relationship for 1964 between the allies' ME/GDP ranks and their GDP ranks, thus supporting the incomeor GDP-based exploitation hypothesis in case 1, where the countries with the larger GDPs shoulder a relatively greater defense burden.

In more recent times, Greece and Turkey spend a greater proportion of their GDP on defense than do many of their richer (higher-GDP) allies in NATO (Sandler and Murdoch 2000). For example, in 2000, Greece and Turkey devoted 3.6 and $3.7 \%$ of their GDPs to military expenditure, respectively, while, e.g., the United Kingdom spent $2.4 \%$ and Germany only $1.5 \%$ of their GDP on ME (SIPRI 2015), which reflects different culturally and historically determined attitudes concerning military activities. In the Greek-Turkish case, traditional grievances between these two countries raised their preferences for defense and motivated their greater burdens compared to their much richer NATO allies. Even though the ME/GDP ratios of these two countries have fallen since then, they are still higher than for most other NATO members (Sandler and George 2016).

In this way, the Greek-Turkish case can be justified by a joint product defense model, in which defense spending gives rise to a country-specific defense benefit and alliance-wide deterrence (Cornes and Sandler 1984; Sandler and Hartley 2001). In this case, the ally's basic utility function remains $u_{i}\left(x_{i}, G\right)$, but in addition there is a private gain from its defense expenditure and thus from its public good contribution. At a NE, an ally equates its weighted sum of marginal rates of substitution from the jointly produced public and private defense outputs to the reciprocal of the price of $G$. Allies with greater private gains from defense have, ceteris paribus, a greater willingness to contribute to the public good. This has been described as an important subcase of case 2 in Sect. 3 above. For now, remarkably, the ME/GDP ratio also is larger for some new NATO members in Eastern Europe, which perceive a stronger foreign threat for historical reasons. According to the World Bank, this ratio has been $1.9 \%$ in Estonia and Poland, and $2.7 \%$ in Lithuania in 2014. Ceteris paribus, larger private defense gains induce an ally to shoulder a larger military expenditure burden in terms of its GDP.

Also for the permanent Western members of the UN Security Council (United States, United Kingdom, and France), ME/GDP are relatively high because these countries have a long-standing tradition of feeling responsible for global security far beyond their own borders and those of their allies. For these new allies and for the UN Security Council members, preferences for defense are stronger than for other members, thereby inducing them to shoulder a larger ME/GDP burden, which reflects the alternative interpretations of the preference-related mechanism of case 2 .

To illustrate case 2 and also case 3 further, we consider the US-Israeli alliance using data from the Stockholm International Peace Research Institute (SIPRI) (2015). Even though Israel has a much smaller GDP than the United States, Israel's ME/GDP exceeded that of the United States as follows: $23 \%$ compared to $7.7 \%$ in 1970, $13.7 \%$ compared to $5.3 \%$ in 1990, and $5.2 \%$ compared to $3.5 \%$ in 2014 (SIPRI 2015). Obviously, Israel has a much stronger preference for defense in the Middle East, insofar as it is surrounded by enemy states, all of which have fought wars with Israel. Moreover, the productivity of Israel in defending itself in the region exceeds that of the United States, which must project its power to the Middle East. In addition, Israel has adapted its weapon systems, even those 
purchased from the United States, to be better suited to the desert battlefields surrounding Israel. Thus, case 3 also comes into play.

Other potential examples exist showing that the mechanisms, which underlie our cases 2 and 3, counteract the classical exploitation hypothesis. Consider peacekeeping as a pure public good that brings stability to the world. In 2000 among NATO allies, Denmark devoted the largest share of its GDP to UN and non-UN-led peacekeeping missions, even though its GDP ranked 13th of the 18 allies (Shimizu and Sandler 2003). The other three highest ranked contributors in 2000 were the Netherlands, Italy, and Norway. Clearly, some smaller-GDP allies - mainly because of specific political attitudes and power relations within these countries-displayed a greater preference for underwriting peacekeeping. Denmark and Norway view peacekeeping as a more desirable public good than do some other NATO allies. Among NATO allies, there is no evidence that some countries' soldiers are better trained at peacekeeping than others, so that case 3 is not germane.

Another field of application of our theoretical results is foreign aid, which is a public good providing nonexcludable and nonrival benefits to countries that care about less fortunate countries. Given Sweden's, Denmark's, and Norway's stated commitment to foreign aid, they provide a much larger share of their GDP to helping poor countries than higherGDP countries, such as the United States (Sandler 2004), so that more than an income effect can be at work. Scandinavia's relatively large contributions to foreign aid are attributable to some specific sense of moral obligation and, hence, abide by case 2 .

Addressing cross-border pollution can also represent cases 2 and 3. In regards to switching from ozone-shield-depleting substances, advanced countries, such as the United States, Japan, and many EU countries, had the advantage over other countries. A productivity edge came from being able to more quickly substitute for ozone-depleting substances with more ozone-benign substitutes (Murdoch and Sandler 1997; Peinhardt and Sandler 2015). This advantage was so great that, consistent with our case 2, the Montreal Protocol not only required greater abatement efforts from these rich countries, but also their underwriting of a Multilateral Fund to assist less capable countries to abide by the treaty (Sandler 2004). Productivity differences with respect to the reduction of sulfur emissions also meant that the Oslo Protocol required larger percentage cutbacks from those countries with a greater productivity in mitigation measures (Finus and Tjøtta 2003). Both of these examples result in an exploitation of the more productive country for the betterment of everyone in ratified international environmental agreements. In this context, we note that these agreements are considered to be mere manifestations of abatement efforts that countries would have undertaken on a voluntary basis (Murdoch and Sandler 1997).

By virtue of a sense of environmental morality (a relatively strong position of the Green Party in its political system), Germany has been more ambitious in its greenhouse gas mitigation efforts than the United States despite German GDP being lower. This outcome reflects case 2. Germany's strong preference for climate protection particularly shows up in its enormous subsidies for renewable energies, which totaled about 24 billion Euros in 2015.

As in case 3, productivity differences also characterize efforts to reduce climate change through a smaller carbon footprint. High-abatement-cost countries typically are reluctant to become active in climate policy. For example, this is the case for countries (such as India, China, and, among the European countries, especially Poland), whose electricity supply heavily depends on the use of coal, which is abundant at low cost so that the opportunity costs of turning to a climate-friendlier energy supply would be high. These carbon-dependent countries have an energy infrastructure that is fossil-fuel oriented, so that to reduce their dependency on these fuels would require tremendous switchover costs. Moreover, as 
seen from the supply-side perspective on climate policy (Sinn 2012), such a switchover also would imply the abandonment of huge resource rents in coal-rich countries.

Of course, some abatement can initially be achieved relatively cheaply through greater efficiency also in carbon-dependent countries, but these abatement costs will rise steeply once these efficiency gains are exhausted and an alternative energy pathway is needed to make steeper carbon cuts. Such a new pathway would be needed for far-reaching decarbonization and the associated deep structural change in energy supply, based either on renewables or nuclear energy or a combination of both. That abatement costs may affect a country's contribution to global climate protection is also corroborated by the recent experience in the United States: Progress in fracking technology and falling costs of shale gas production made it possible to substitute less carbon-intensive natural gas for dirty coal. This is considered to be a major cause for the reduction in greenhouse gas emissions in the United States, which fell by $9 \%$ between 2005 and 2013 (Environmental Protection Agency (EPA) 2015).

Moreover, some countries have a productivity advantage in terms of the use of renewable energy sources. In the United States, Texas, Hawaii, and Wyoming have an advantage over many other states in the production of wind power owing to wind patterns, while coastal states will have an advantage in wave-produced power as the technology is further developed. Sun-drenched states, such as Arizona, Nevada, and Texas potentially have an advantage in the production of solar energy. In Europe, e.g., Denmark and Northern Germany have an advantage in wind power, whereas the southern European countries (such as Spain, Italy, and Greece) enjoy lower costs in generating solar energy. One might expect that these comparative advantages will foster contributions to the global public good of climate protection. Moreover, country-specific ancillary benefits of greenhouse gas abatement will also increase a country's willingness to carry out corresponding measures (Finus and Rübbelke 2013), which also is captured by case 2 .

Acknowledgments Wolfgang Buchholz gratefully acknowledges financial support from the German Federal Ministry of Education and Research BMBF through the research project ECCUITY (FKZ 01LA1104B) as part of the funding priority "Economics of Climate Change". Todd Sandler received support from the Vibhooti Shukla endowment at the University of Texas at Dallas. We also want to thank a referee for many constructive and-thought-provoking comments, which helped improve the paper considerably.

Open Access This article is distributed under the terms of the Creative Commons Attribution 4.0 International License (http://creativecommons.org/licenses/by/4.0/), which permits unrestricted use, distribution, and reproduction in any medium, provided you give appropriate credit to the original author(s) and the source, provide a link to the Creative Commons license, and indicate if changes were made.

\section{References}

Andreoni, J. (1988). Privately provided public goods in a large economy: The limits of altruism. Journal of Public Economics, 35(1), 57-73.

Andreoni, J. (1990). Impure altruism and donations to public goods: A theory of warm-glow giving. Economic Journal, 100(401), 464-477.

Andreoni, J., \& McGuire, M. C. (1993). Identifying the free riders: A simple algorithm for determining who will contribute to a public good. Journal of Public Economics, 51(3), 447-454.

Bergstrom, T. C., Blume, L., \& Varian, H. (1986). On the private provision of public goods. Journal of Public Economics, 29(1), 25-49.

Boadway, R., \& Hayashi, M. (1999). Country size and the voluntary provision of international public goods. European Journal of Political Economy, 15(4), 619-638. 
Buchholz, W., Cornes, R., \& Rübbelke, D. (2014). Potentially harmful international cooperation on global public good provision. Economica, 81(2), 205-223.

Buchholz, W., \& Konrad, K. A. (1994). Global environmental problems and the strategic choice of technology. Journal of Economics, 60(3), 299-321.

Cornes, R., \& Hartley, R. (2007). Aggregative public good games. Journal of Public Economic Theory, 9(2), 201-219.

Cornes, R., \& Sandler, T. (1984). Easy riders, joint production, and public goods. Economic Journal, 94(3), 580-598.

Cornes, R., \& Sandler, T. (1994). The comparative static properties of the impure public good model. Journal of Public Economics, 54(3), 403-421.

Cornes, R., \& Sandler, T. (1996). The theory of externalities, public goods, and club goods (2nd ed.). Cambridge: Cambridge University Press.

Environmental Protection Agency (EPA) (2015). Climate change indicators in the United States: U.S. greenhouse gas emissions. Accessed 7 June 2015. https://www3.epa.gov/climatechange/science/ indicators/ghg/us-ghg-emissions.html.

Finus, M., \& Rübbelke, D. T. G. (2013). Public good provision and ancillary benefits: The case of climate agreements. Environmental \& Resource Economics, 56(2), 211-226.

Finus, M., \& Tjøtta, S. (2003). Oslo Protocol and sulphur reduction: The great leap forward. Journal of Public Economics, 87(9-10), 2031-2048.

Fraser, C. D. (1992). The uniqueness of Nash equilibrium in the private provision of public goods. Journal of Public Economics, 49(3), 389-390.

Ihori, T. (1996). International public goods and contribution productivity differentials. Journal of Public Economics, 61(1), 139-154.

Ihori, T., McGuire, M. C., \& Nakagawa, S. (2014). International security, multiple public good provision, and the exploitation hypothesis. Defence and Peace Economics, 25(3), 213-229.

Kotchen, M. J. (2007). Equilibrium existence and uniqueness in impure public good models. Economics Letters, 97(2), 91-96.

Murdoch, J., \& Sandler, T. (1997). The voluntary provision of a pure public good: The case of reduced CFC emissions and the Montreal Protocol. Journal of Public Economics, 63(2), 331-349.

Olson, M. (1965). The logic of collective action. Cambridge, MA: Havard University Press.

Olson, M., \& Zeckhauser, R. (1966). An economic theory of alliances. The Review of Economics and Statistics, 48(3), 266-279.

Pecorino, P. (2015). Olson's Logic of Collective Action at fifty. Public Choice, 162(3-4), $243-262$.

Peinhardt, C., \& Sandler, T. (2015). Transnational cooperation: An issue-based approach. New York: Oxford University Press.

Pittel, K., \& Rübbelke, D. T. G. (2008). Climate policy and ancillary benefits: A survey and integration into the modeling of international negotiations on climate change. Ecological Economics, 68(1-2), $210-220$.

Sandler, T. (1992). Collective action: theory and applications. Ann Arbor, MI: University of Michigan Press.

Sandler, T. (2004). Global collective action. Cambridge: Cambridge University Press.

Sandler, T. (2015). Collective action: Fifty years later. Public Choice, 164(3-4), 195-216.

Sandler, T., \& George, J. (2016). Military expenditure trends for 1960-2014 and what they reveal. Global Policy, 7(2), 174-184.

Sandler, T., \& Hartley, K. (2001). Economic of alliances: The lessons for collective action. Journal of Economic Literature, 39(3), 869-896.

Sandler, T., \& Murdoch, J. C. (2000). On sharing NATO defence burdens in the 1990s and beyond. Fiscal Studies, 21(3), 297-327.

Shimizu, H., \& Sandler, T. (2003). NATO peacekeeping and burden sharing, 1994-2000. Public Finance Review, 31(2), 123-143.

Sinn, H.-W. (2012). The green paradox: A supply-side approach to global warming. Cambridge, MA: The MIT Press.

Stockholm International Peace Research Institute (SIPRI). (2015). SIPRI extended military expenditure database, preliminary version. Stockholm: SIPRI. 